\title{
TOPOLOGICAL OBJECTS AND SHEAVES
}

\author{
BY \\ YEATON H. CLIFTON(1) AND J. WOLFGANG SMITH(2)
}

In this paper we shall be concerned with a category $\mathscr{C}$, previously introduced in [2]. We recall that the objects and maps in $\mathscr{C}$ (henceforth referred to simply as objects and maps) constitute a generalization of the concepts of topological space and continuous function. In particular, one finds that the category $C$ of topological spaces and continuous functions may be canonically identified with a (proper) subcategory $\mathfrak{C}$ of $\mathscr{C}$. Moreover, it has been shown in [2] that every subcategory $C_{0}$ of $C$ admits a canonical extension to a subcategory $\mathscr{C}_{0}$ of $\mathscr{C}$, which in some sense is maximal. It may now be asked whether classical theories, defined on a subcategory $C_{0}$ of $C$, admit a natural extension to the corresponding object category $\mathscr{C}_{0}$. This extension problem has been considered in [2] for the case of theories arising from a sheaf-valued functor. In the present paper we will describe a canonical extension of such functors in full detail, and shall establish pertinent results.

Some effort has been made in [2] to indicate the original motivation which has led to the definition of object categories. It has been pointed out in this connection that numerous structures (e.g., Lie groups, homogeneous spaces, foliated or affinely connected manifolds) give rise to canonical objects. The program of extending concepts of topology and geometry to object categories is therefore suggestive of various applications.

At this point it seems appropriate to review in broad terms what is meant by an object, and in what respect a general object differs fundamentally from a topological space. An object $\mathscr{X}$ has been conceived as a pair $(X, I)$, where $X$ is a topological space and $I$ a collection of local maps $f: X \rightarrow X$, subject to certain axioms. Given two objects $\mathscr{X}$ and $\mathscr{X}^{\prime}$, one conceives a map $F: \mathscr{X} \rightarrow \mathscr{X}^{\prime}$ as a collection of local maps $f: X \rightarrow X^{\prime}$, subject again to appropriate axioms. Having defined the composition of maps, one arrives at the category $\mathscr{C}$. At this stage one observes that a considerable part of the structure which enters into the definition of an object $\mathscr{X}$ is not preserved under $\mathscr{C}$-isomorphism. On the other hand, we have adopted the viewpoint that only those properties of $(X, I)$ which are

Received by the editors October 4, 1961 .

(1) The work of this author has been supported in part by the National Science Foundation.

(2) This research was supported in part by the United States Air Force under contract No. AF-49(638)-42, monitored by the Air Force Office of Scientific Research of the Air Research and Development Command. 
invariant under isomorphism will be regarded as intrinsic properties of $\mathscr{X}$. To bring out this point more explicitly, one could have defined the object $\mathscr{X}$ as the equivalence class of $(X, I)$ in $\mathscr{C}$ under isomorphism, in which case $(X, I)$ would appear as one among many possible representatives of $\mathscr{X}$. Thus $\mathscr{X}$ could have a second representative $\left(X^{\prime}, I^{\prime}\right)$ such that $X$ and $X^{\prime}$ are not homeomorphic, or even equipotent. We have not introduced this formal distinction between the object $\mathscr{X}$ and a representative $(X, I)$, on the grounds that the distinction is unnecessarily cumbersome. However, it needs to be emphasized that the essential character of an object derives from our concept of object map and object isomorphism. It is this circumstance which distinguishes an object $\mathscr{X}$ from the pair $(X, I)$, conceived of in the ordinary way.

We will begin in $\S 1$ by establishing the basic theory of category $\mathscr{C}$. In contradistinction to the language used in [2], we shall employ sheaf language in order to effect a clear separation between the algebraic and topological aspects of the theory. It should be pointed out, however, that the less abstract language of [2] may be found more suggestive to the intuition. The actual extension of sheaf-valued functors to object categories will be carried out in $\$ 2$. As previously noted in [2], this extension gives rise to some new cohomology functors, these being trivial on the original category $\mathscr{C}_{0}$.

Following the convention adopted by some writers, we shall often treat categories as if they were sets. This calls for a word of caution. It is recognized, for instance, that the class of all topological spaces cannot be regarded as a set. To avoid the possibility of antinomies, we consequently recommend that the categories in question be regarded as specific collections of objects and maps, which are somehow given.

1. Fundamental concepts. 1.1. Notation. Let $X$ and $X^{\prime}$ be topological spaces. By a local map $f: X \rightarrow X^{\prime}$ we shall understand a continuous function whose domain is an open subset of $X$ and which takes values in $X^{\prime}$. We shall let $C_{X, X^{\prime}}$ denote the sheaf(3) over $X$ of local maps $f: X \rightarrow X^{\prime}$, and we let $G_{X, X^{\prime}}$ denote the corresponding espace étalé(4). We recall that $C_{X, X^{\prime}}$ is a function which, to every open subset $U$ of $X$, assigns the set $C\left(U, X^{\prime}\right)$ of all maps $f: U \rightarrow X^{\prime}$, and to every pair $(U, V)$ of such open subsets with $V \subset U$, assigns the restriction map $\rho: C\left(U, X^{\prime}\right) \rightarrow C\left(V, X^{\prime}\right)$. The space $G_{X, X^{\prime}}$ is then the set of all germs of local maps $f: X \rightarrow X^{\prime}$, endowed with the appropriate topology. The set of all germs arising from a given local map $f$ constitutes an open subset of $G_{X, X^{\prime}}$, which will be denoted by $|f|$. We will let $D: G_{X, X^{\prime}} \rightarrow X$ denote the natural projection, and Im: $G_{X, X^{\prime}} \rightarrow X^{\prime}$ denote the function which to every germ $\gamma \in G_{X, X^{\prime}}$ assigns its image point in $X^{\prime}$. We observe now that the composition product of functions determines a partially defined multiplication of germs. More specifically, two

(3) Godement [3, p. 109].

(4) Op. cit., p. 110. 
germs $\gamma \in G_{\boldsymbol{X}, \boldsymbol{X}^{\prime}}$ and $\gamma^{\prime} \in G_{\boldsymbol{X}^{\prime}, \boldsymbol{X}^{\prime \prime}}$ with $\operatorname{Im}(\gamma)=D\left(\gamma^{\prime}\right)$ determine a germ in $G_{\boldsymbol{X}, \boldsymbol{X}^{\prime \prime}}$, which we shall denote by $\gamma^{\prime} \gamma$. Lastly, if $X$ is a topological space, $i_{X}$ shall denote the identity map of $X$.

1.2. Topological OBJECTS. It will be convenient to separate the axioms for a topological object into two parts, as follows:

Definition 1. A preobject is a pair $(X, J)$, where $X$ is a topological space and $J$ is an open subset of $G_{X, X}$ which satisfies the following extension axiom: Given $\alpha, \beta \in J$ with $D(\alpha)=D(\beta)$, there shall exist $\bar{\alpha}, \bar{\beta} \in J$ such that $\bar{\alpha} \alpha=\bar{\beta} \beta$.

Definition 2. A topological object (henceforth referred to as an object) is a preobject $(X, I)$ such that $\left|i_{X}\right| \subset I$, and satisfying the following closure axiom: Given $\bar{\alpha}, \beta, \bar{\beta} \in I$ and $\alpha \in G_{X, X}$ such that $\bar{\alpha} \alpha=\bar{\beta} \beta$, then $\alpha \in I$.

An object $(X, I)$ is said to be generated by $(X, J)$, where $J$ is a subset of $G_{X, X}$, if $J \subset I$, and if further, for every object $\left(X, I^{\prime}\right)$ with $J \subset I^{\prime}, I$ is a subset of $I^{\prime}$. It follows that at most one object can be generated by $(X, J)$. We shall now prove

Proposition 1. A preobject $(X, J)$ generates an object $(X, I)$. Moreover, $I$ is the set of all germs $\alpha \in G_{X X}$ such that $\bar{\alpha} \alpha=\bar{\beta}$, where $\bar{\alpha}$ and $\bar{\beta}$ are finite products of germs in $J \cup\left|i_{X}\right|$.

Given a preobject $(X, J)$, we set $J_{1}=J \cup\left|i_{X}\right|$. Since $J$ and $\left|i_{X}\right|$ are both open in $G_{X, X}$, so is $J_{1}$. It is also easy to see that $J_{1}$ satisfies the extension axiom. Consequently $\left(X, J_{1}\right)$ is again a preobject.

Now let $J_{2}$ denote the set of all germs $\alpha \in G_{X, X}$ which are of the form

$$
\alpha=\alpha_{n} \cdots \alpha_{2} \alpha_{1}
$$

where $n$ is a positive integer and $\alpha_{1}, \alpha_{2}, \cdots, \alpha_{n} \in J_{1}$. We assert that $\left(X, J_{2}\right)$ is a preobject. In the first place $J_{2}$ is open, for if $\alpha$ is given as above, there exist local maps $f_{1}, f_{2}, \cdots, f_{n}: X \rightarrow X$ such that (i) $\alpha_{i} \in\left|f_{i}\right| \subset J_{1}(1 \leqq i \leqq n)$; (ii) the product $f=f_{n} \circ \cdots \circ f_{2} \circ f_{1}$ is defined. Then $|f| \subset J_{2}$ and $|f|$ is a neighborhood of $\alpha$ in $G_{X}, \boldsymbol{X}$.

It remains to show that $J_{2}$ satisfies the extension axiom. For this purpose we consider two elements $\alpha=\alpha_{n} \cdots \alpha_{2} \alpha_{1}$ and $\beta=\beta_{m} \cdots \beta_{2} \beta_{1}$ in $J_{2}$, the $\alpha_{i}$ and $\beta_{j}$ being elements of $J_{1}$. If $D(\alpha)=D(\beta)$, then $D\left(\alpha_{1}\right)=D\left(\beta_{1}\right)$, and since $J_{1}$ satisfies the extension axiom, one concludes that there exist germs $\delta_{11}, \gamma_{11} \in J_{1}$ such that $\gamma_{11} \alpha_{1}=\delta_{11} \beta_{1}$. Proceeding in this manner, one obtains the commutative diagram on the next page. Consequently $\bar{\alpha} \alpha=\bar{\beta} \beta$, with $\bar{\alpha}=\gamma_{n m} \cdots \gamma_{n 2} \gamma_{n 1}$ and $\bar{\beta}=\delta_{n m} \cdots \delta_{2 m} \delta_{1 m}$, as was to be shown.

Finally, we let $I$ denote the set of all germs $\alpha \in G_{X, X}$ such that

$$
\alpha^{*} \alpha=\gamma \text { for some } \alpha^{*}, \gamma \in J_{2} \text {. }
$$

We assert that $(X, I)$ is an object. In the first place $I$ is open in $G_{X, X}$, for if $\alpha$ satisfies condition (1.2), there exist local maps $f, f^{*}, g: X \rightarrow X$ such that (i) $\alpha \in|f|$, 

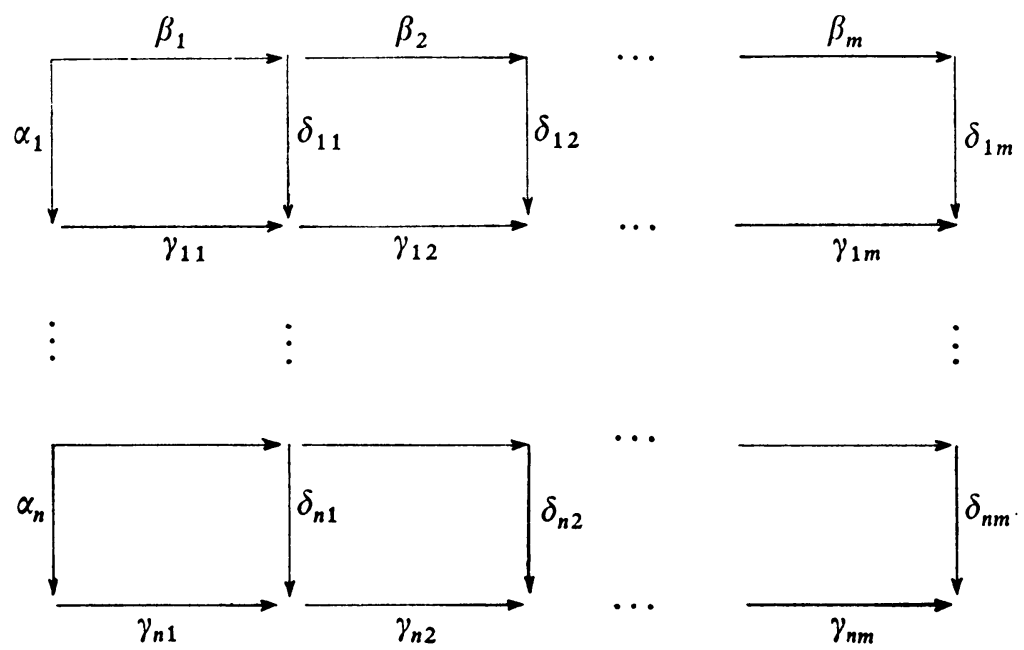

$\alpha^{*} \in\left|f^{*}\right| \subset J_{2}$ and $\gamma \in|g| \subset J_{2}$; (ii) $f^{*} \circ f=g$. Then $|f| \subset I$ and $|f|$ is a neighborhood of $\alpha$ in $G_{X, X}$.

We show next that $I$ satisfies the extension axiom. Let $\alpha, \beta \in I$ with $D(\alpha)=D(\beta)$ By definition of $I$, there exist $\alpha^{*}, \beta^{*}, \gamma, \delta \in J_{2}$ such that $\alpha^{*} \alpha=\gamma$ and $\beta^{*} \beta=\delta$. Since $\left(X, J_{2}\right)$ is a preobject, there exist $\bar{\gamma}, \bar{\delta} \in J_{2}$ such that $\bar{\gamma} \gamma=\bar{\delta} \delta$. Hence $\bar{\alpha} \alpha=\bar{\beta} \beta$. where $\bar{\alpha}=\bar{\gamma} \alpha^{*}$ and $\bar{\beta}=\bar{\delta} \beta^{*}$, as was to be shown.

We now note that $\left|i_{X}\right| \subset I$. To prove that $(X, I)$ is an object, it therefore remains to verify that $I$ satisfies the closure axiom. Let $\alpha \in G_{X, X}$ and $\bar{\alpha}, \beta, \bar{\beta} \in I$ be given, with $\bar{\alpha} \alpha=\bar{\beta} \beta$. Then there exist $\bar{\alpha}^{*}, \beta^{*}, \bar{\beta}^{*}, \bar{\gamma}, \delta, \bar{\delta} \in I$ such that $\bar{\alpha}^{*} \bar{\alpha}=\bar{\gamma}$, $\beta^{*} \beta=\delta$ and $\bar{\beta}^{*} \bar{\beta}=\bar{\delta}$. Since $J_{2}$ satisfies the extension axiom, it is apparent from diagram (1.3) that the germs indicated by the additional arrows all exist and make the diagram commutative.

Hence $\alpha^{*} \alpha=\gamma$, with $\alpha^{*}=\gamma_{2} \gamma_{1} \bar{\gamma}$ and $\gamma=\delta_{2} \delta_{1} \delta$. We conclude that $(X, I)$ is an object.

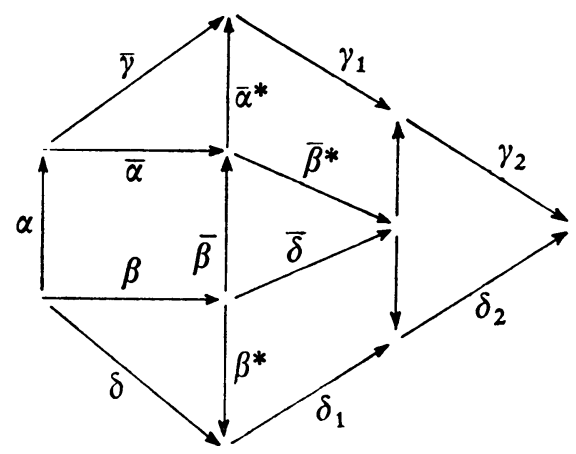


It is clear from our construction that $J \subset J_{1} \subset J_{2} \subset I$. It is likewise evident that $I$ contains no unnecessary elements, which implies that if $\left(X, I^{\prime}\right)$ is an object with $J \subset I^{\prime}$, then $I \subset I^{\prime}$. Consequently $(X, J)$ generates $(X, I)$, as was to be proved.

1.3. OBJECT MAPS. One now requires a concept of "map" $F: \mathscr{X} \rightarrow \mathscr{X}^{\text {' when }}$ $\mathscr{X}$ and $\mathscr{X}^{\prime}$ are objects. Again we shall separate the definition into two parts, as follows:

Definition 3. Let $\mathscr{X}=(X, I)$ and $\mathscr{X}^{\prime}=\left(X^{\prime}, I^{\prime}\right)$ be objects. A premap $F$ : $\mathscr{X} \rightarrow \mathscr{X}^{\prime}$ is an open subset of $G_{X, X^{\prime}}$ such that $D(F)=X$, and satisfying the following extension axiom: Given $\alpha \in I$ and $\phi, \psi \in F$ with $D(\alpha)=D(\phi)$ and $\operatorname{Im}(\alpha)=$ $D(\psi)$, there shall exist $\alpha^{\prime}, \beta^{\prime} \in I^{\prime}$ such that $\beta^{\prime} \phi=\alpha^{\prime} \psi \alpha$.

Definition 4. A map $F: \mathscr{X} \rightarrow \mathscr{X}^{\prime}$ is a premap satisfying the following closure axiom: Given $\alpha \in I, \phi \in G_{X, X^{\prime}}, \psi \in F$ and $\alpha^{\prime}, \beta^{\prime} \in I^{\prime}$ such that $\beta^{\prime} \phi=\alpha^{\prime} \psi \alpha$, then $\phi \in F$.

As an immediate consequence of these definitions, one has

Proposition 2. Let $\mathscr{X}=(X, I), \mathscr{X}^{\prime}=\left(X^{\prime}, I^{\prime}\right)$ be objects and $F, G: \mathscr{X} \rightarrow \mathscr{X}^{\prime}$ be maps. If $F \subset G$, then $F=G$.

For let $\phi \in G$. Since $D(F)=X$, there exists a germ $\psi \in F$ such that $D(\psi)=D(\phi)$. Since $G$ satisfies the extension axiom, there exist $\alpha^{\prime}, \beta^{\prime} \in I^{\prime}$ such that $\beta^{\prime} \phi=\alpha^{\prime} \psi\left({ }^{5}\right)$. But this implies by the closure axiom that $\phi \in F$. Hence $G \subset F$, as was to be proved.

Concerning the connection between premaps and maps, we shall now prove

Proposition 3. Let $\mathscr{X}=(X, I), \mathscr{X}^{\prime}=\left(X^{\prime}, I^{\prime}\right)$ be objects, and let $G$ be an open subset of $G_{X, X}$ such that $D(G)=X$. Then $G$ is a premap if and only if there exists a map $F: \mathscr{X} \rightarrow \mathscr{X}^{\prime}$ with $G \subset F$. Moreover, $F$ is unique.

In the first place it is clear that if $F$ is a map and $G \subset F$, then $G$ will satisfy the extension axiom, because $F$ does. Hence if $F$ exists, then $G$ is a premap. Conversely, we suppose now that $G: \mathscr{X} \rightarrow \mathscr{X}^{\prime}$ is a premap. Let $F$ denote the set of all germs $\phi \in G_{X, X^{\prime}}$ such that $\beta^{\prime} \phi=\alpha^{\prime} \psi \alpha$ for some $\alpha \in I, \psi \in G$ and $\alpha^{\prime}, \beta^{\prime} \in I^{\prime}$. Clearly $G \subset F$. We assert further that $F: \mathscr{X} \rightarrow \mathscr{X}^{\prime \prime}$ is a map. To verify this, one first observes that $F$ is open in $G_{X, X^{\prime}}$, as follows by the type of reasoning described in the proof of Proposition 1. To show that $F$ satisfies the extension axiom, we let $\alpha \in I$ and $\phi, \psi \in F$ with $D(\alpha)=D(\phi)$ and $\operatorname{Im}(\alpha)=D(\psi)$. We consider the diagram on the following page:

The germs $\alpha_{i} \in I, \psi_{i} \in G$ and $\alpha_{i}^{\prime}, \beta_{i}^{\prime} \in I^{\prime}(i=1,2)$ exist by definition of $F$. Moreover, since $D(G)=X$, there exist $\sigma, \tau \in G$ with $D(\sigma)=D(\alpha)$ and $D(\tau)=D(\phi)$. Germs indicated by the remaining arrows in diagram (1.4) all belong to $I^{\prime}$, and they exist because $G$ and $I^{\prime}$ satisfy respective extension axioms. It follows now from the commutativity of the diagram that $\beta^{\prime} \phi=\alpha^{\prime} \psi \alpha$, with $\alpha^{\prime}=\gamma_{4} \gamma_{3} \gamma_{2} \gamma_{1} \beta_{2}^{\prime}$ and $\beta^{\prime}=\gamma \beta_{1}^{\prime}$.

(5) At this point it must be recalled that $\left|i_{X^{\prime}}\right| \subset I^{\prime}$. 


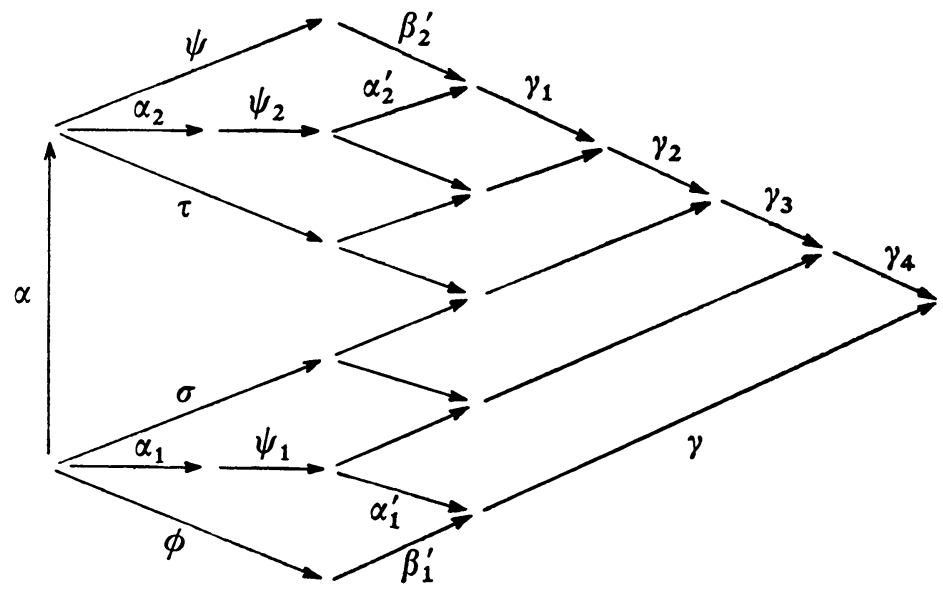

It remains to show that $F$ satisfies the closure axiom. For this purpose we consider the diagram:

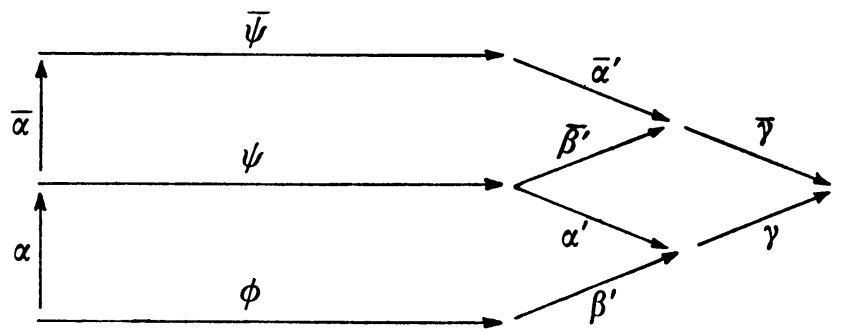

where $\alpha \in I, \phi \in G_{X, X^{\prime}}, \psi \in F$ and $\alpha^{\prime}, \beta^{\prime} \in I^{\prime}$ are given initially. Then $\bar{\alpha} \in I, \bar{\psi} \in F$ and $\bar{\alpha}^{\prime}, \bar{\beta}^{\prime} \in I^{\prime}$ exist by definition of $F$, and $\bar{\gamma}, \gamma$ exist by the extension axiom for $I^{\prime}$. From the commutativity of the diagram we can conclude that $\phi \in F$, as was to be shown.

Hence, if $G$ is a premap, there exists a map $F$ with $G \subset F$. It follows from our construction of $F$ that any other map $F^{\prime}: \mathscr{X} \rightarrow \mathscr{X}^{\prime}$ with $G \subset F^{\prime}$ must contain $F$. Uniqueness follows now by Proposition 2. q.e.d.

Our next task shall be to define the composition of object maps. For this purpose one requires the following

Lemma. Let $F: \mathscr{X} \rightarrow \mathscr{X}^{\prime}$ and $F^{\prime}: \mathscr{X}^{\prime} \rightarrow \mathscr{X}^{\prime \prime}$ be maps, and let $H$ denote the set of all germs $\psi \in G_{X, X^{\prime \prime}}$ such that $\psi=\phi^{\prime} \phi$ for some $\phi \in F$ and $\phi^{\prime} \in F^{\prime}$. Then $H$ is a premap.

The proof follows easily by methods familiar from the preceding paragraphs, and will be omitted here. On the strength of this lemma, together with Proposition 3 , there exists a unique map from $\mathscr{X}$ to $\mathscr{X}$ " containing $H$. We shall call it the composite of $F$ by $F^{\prime}$, and denote it by $F^{\prime} \circ F$. 
As an easy consequence of our definitions, we now state

Proposition 4. Let $\mathscr{X}=(X, I)$ be an object. Then $I: \mathscr{X} \rightarrow \mathscr{X}$ is a map. If $\mathscr{X}^{\prime}=\left(X^{\prime}, I^{\prime}\right)$ is a second object and $F: \mathscr{X} \rightarrow \mathscr{X}^{\prime}$ is a map, then $F \circ I=I^{\prime} \circ F=F$.

Given an object $\mathscr{X}=(X, I)$, we may consequently refer to $I$ as the identity map of $\mathscr{X}$.

1.4. Categories. We now proceed to show that the concepts introduced in $\S \S 1.2$ and 1.3 give rise to a category $\mathscr{C}$ which extends the category $C$ of topological spaces and continuous functions. To make this precise, we will use the language of Cartan and Eilenberg $[1]\left({ }^{6}\right)$.

Definition 5. Given two objects $\mathscr{X}, \mathscr{X}^{\prime}$; let $\operatorname{Hom}_{\mathscr{C}}\left(\mathscr{X}, \mathscr{X}^{\prime}\right)$ denote the set of all maps $F: \mathscr{X} \rightarrow \mathscr{X}^{\prime}$. Let $\mathscr{C}$ denote the triple consisting of

(i) the class of objects $\mathscr{X}, \mathscr{X}^{\prime}, \cdots$;

(ii) the function which to each pair $\left(\mathscr{X}, \mathscr{X}^{\prime}\right)$ of objects assigns the set $\operatorname{Hom}_{\mathscr{C}}\left(\mathscr{X}, \mathscr{X}^{\prime}\right)$;

(iii) the function $\circ$ from $\operatorname{Hom}_{\mathscr{C}}\left(\mathscr{X}, \mathscr{X}^{\prime}\right) \times \operatorname{Hom}_{\mathscr{C}}\left(\mathscr{X}^{\prime}, \mathscr{X}^{\prime \prime}\right)$ into $\operatorname{Hom}_{\mathscr{C}}\left(\mathscr{X}, \mathscr{X}^{\prime \prime}\right)$, defined for every triple $\left(\mathscr{X}, \mathscr{X}^{\prime}, \mathscr{X}^{\prime \prime}\right)$ of objects, which to every element $\left(F, F^{\prime}\right) \in$ $\operatorname{Hom}_{\mathscr{C}}\left(\mathscr{X}, \mathscr{X}^{\prime}\right) \times \operatorname{Hom}_{\mathscr{C}}\left(\mathscr{X}^{\prime}, \mathscr{X}^{\prime \prime}\right)$ assigns the element $F^{\prime} \circ F \in \operatorname{Hom}_{\mathscr{C}}\left(\mathscr{X}, \mathscr{X}^{\prime \prime}\right)$.

THEOREM 1. $\mathbb{C}$ is a category. Moreover, $C$ can be naturally identified with a full subcategory $\mathfrak{C}$ of $\mathscr{C}$.

To show that $\mathscr{C}$ is a category, one must verify two axioms, the first of which asserts that composition of maps is associative. To check this, we consider three maps $F_{i}: \mathscr{X}_{i} \rightarrow \mathscr{X}_{i+1}(i=1,2,3)$, where $\mathscr{X}_{j}=\left(X_{j}, I_{j}\right)$ are objects. Let $H$ denote the set of all germs $\psi \in G_{X_{1}, X_{4}}$ such that $\psi=\phi_{3} \phi_{2} \phi_{1}$ for some $\phi_{i} \in F_{i}$. It follows immediately that $H$ is open in $G_{X_{1}, X_{4}}$, that $D(H)=X_{1}$, and that $H \subset F_{3} \circ\left(F_{2} \circ F_{1}\right)$ and $H \subset\left(F_{3} \circ F_{2}\right) \circ F_{1}$. Hence $F_{3} \circ\left(F_{2} \circ F_{1}\right)=\left(F_{3} \circ F_{2}\right) \circ F_{1}$ by Proposition 3 (both equal the unique map generated by $H$ ). The second category axiom pertains to the existence of identity maps, and is implied by Proposition 4. Hence $\mathscr{C}$ is a category.

Now let $\mathscr{I}$ denote the function which to every topological space $X$ assigns the object $\mathscr{X}_{X}=\left(X,\left|i_{X}\right|\right)$, and to every continuous function $f: X \rightarrow X^{\prime}$ assigns the object map $F_{f}=|f|: \mathscr{X}_{X} \rightarrow \mathscr{X}_{X^{\prime}}$. One verifies easily that $\mathscr{I}$ is a (covariant) functor from $C$ to $\mathscr{C}$. Let $\mathbb{C}$ denote the image of $C$ under $\mathscr{I}$. Then $\mathbb{C}$ is a subcategory of $\mathscr{C}$, and it is also clear that $\mathscr{I}$ establishes an isomorphism of $C$ with $\mathscr{C}$. It remains therefore to show that $\mathbb{C}$ is a full subcategory of $\mathscr{C}$, i.e. that $\operatorname{Hom}_{\mathscr{C}}\left(\mathscr{X}_{X}, \mathscr{X}_{X^{\prime}}\right) \subset \operatorname{Hom}_{\mathbb{C}}\left(\mathscr{X}_{X}, \mathscr{X}_{X^{\prime}}\right)$ for arbitrary objects $\mathscr{X}_{X}, \mathscr{X}_{X^{\prime}}$ in $\mathbb{C}$. For this purpose we consider an arbitrary map $F: \mathscr{X}_{X} \rightarrow \mathscr{X}_{X^{\prime}}$. If $\phi, \psi \in F$ and $D(\phi)=D(\psi)$, then we may conclude by the extension axiom for maps that there exist $\alpha^{\prime}, \beta^{\prime} \in\left|i_{X^{\prime}}\right|$ such that $\alpha^{\prime} \phi=\beta^{\prime} \psi$. But this means that $\phi=\psi$. Consequently $F$ is the image

(6) A considerable part of this terminology is also found in Godement [3, pp. 11-13]. 
of $X$ under a cross-section $\sigma: X \rightarrow G_{X, X^{\prime}}$. One sees now that $\sigma$ corresponds to a continuous function $f: X \rightarrow X^{\prime}$, and that $F=|f|$ as was to be proved.

Given a subcategory $C_{0}$ of $C$, we will let $\mathfrak{C}_{0}$ denote the subcategory of $\mathfrak{C}$ which corresponds to $C_{0}$ under the identification functor $\mathscr{I}$. We proceed to construct a canonical extension of $\mathfrak{C}_{0}$ to a subcategory $\mathscr{C}_{0}$ of $\mathscr{C}$. For every pair $\left(X, X^{\prime}\right)$ of spaces in $C_{0}$, we let $G_{X, X^{\prime}}^{0}$ denote the set of all germs $\phi \in G_{X, X^{\prime}}$ such that $\phi \in|f|$ for some map $f$ in $C_{0}$. For every object $(X, I)$ with $X$ in $C_{0}$, we let $I_{0}=I \cap G_{X, X}^{0}$. Let $\mathscr{B}_{0}$ denote the class of objects $\mathscr{X}=(X, I)$ such that $X$ is in $C_{0}$ and $\left(X, I_{0}\right)$ is a preobject generating $\mathscr{X}$. Given a map $F: \mathscr{X} \rightarrow \mathscr{X}^{\prime}$ with $\mathscr{X}=(X, I)$ and $\mathscr{X}^{\prime}=\left(X^{\prime}, I^{\prime}\right)$ in $\mathscr{B}_{0}$, we let $F_{0}=F \cap G_{X, X^{\prime}}^{0}$. Lastly, for every pair $\left(\mathscr{X}, \mathscr{X}^{\prime}\right)$ of objects in $\mathscr{B}_{0}$, we let $\operatorname{Hom}_{\mathscr{C}_{0}}\left(\mathscr{X}, \mathscr{X}^{\prime}\right)$ denote the set of all maps $F: \mathscr{X} \rightarrow \mathscr{X}^{\prime}$ such that $F_{0}$ is a premap. Clearly $F \in \operatorname{Hom}_{\mathscr{C}_{0}}\left(\mathscr{X}, \mathscr{X}^{\prime}\right)$ and $F^{\prime} \in$

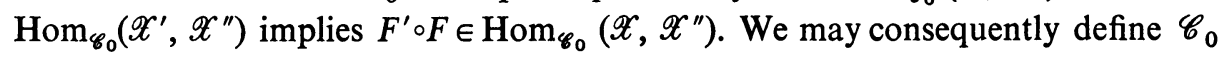
to be the triple consisting of

(i) the class $\mathscr{B}_{0}$;

(ii) the function which to each pair $\left(\mathscr{X}, \mathscr{X}^{\prime}\right)$ of objects in $\mathscr{B}_{0}$ assigns the set $\operatorname{Hom}_{\mathscr{C}_{0}}\left(\mathscr{X}, \mathscr{X}^{\prime}\right)$;

(iii) the function $\circ$, restricted to sets $\operatorname{Hom}_{\mathscr{C}_{0}}\left(\mathscr{X}, \mathscr{X}^{\prime}\right) \times \operatorname{Hom}_{\mathscr{C}_{0}}\left(\mathscr{X}^{\prime}, \mathscr{X}^{\prime \prime}\right)$, with $\mathscr{X}, \mathscr{X}^{\prime}$ and $\mathscr{X}^{\prime \prime}$ in $\mathscr{B}_{0}$.

Proposition 5. $\mathscr{C}_{0}$ is a subcategory of $\mathscr{C}$. Moreover, $\mathfrak{C}_{0}$ is a full subcategory of $\mathscr{C}_{0}$.

The proof is straightforward and can be omitted.

It should be noted that an object in $\mathscr{C}_{0}$ which is isomorphic in $\mathscr{C}_{0}$ to an object in $\mathfrak{C}_{0}$ may not itself belong to $\mathfrak{C}_{0}$. One may refer to such objects as quasi-spaces of type $C_{0}$.

1.5. THE BASIC SPACE OF AN OBJECT. In the following paragraphs we shall construct a natural (covariant) functor $\mathscr{P}: \mathscr{C} \rightarrow \mathscr{C}$, together with a morphism $\left({ }^{7}\right)$ $P$ from the identity functor of $\mathscr{C}$ to $\mathscr{P}$, which play an important role in the theory of objects. The functor $\mathscr{P}$, in particular, gives rise to a local category structure on $\mathscr{C}$. To define $P$ and $\mathscr{P}$, we associate with every object $\mathscr{X}=(X, I)$ the set $Q$ of pairs $(x, y) \in X \times X$ such that $D(\alpha)=x, D(\beta)=y$ and $\operatorname{Im}(\alpha)=\operatorname{Im}(\beta)$ for some $\alpha, \beta \in I$. It is immediate that $Q$ is reflexive and symmetric, and by the extension axiom for objects, $Q$ is also transitive. Let $\hat{X}$ denote the set of equivalence classes $\bmod Q$ in $X$, endowed with the quotient topology, and let $p_{x}: X \rightarrow \hat{X}$ denote the natural projection. We assert that $\left|p_{\mathscr{X}}\right|: \mathscr{X} \rightarrow \mathscr{I}(\hat{\mathscr{X}})$ is a premap. Since clearly $D\left(\left|p_{x}\right|\right)=X$, one need only verify that $\left|p_{x}\right|$ satisfies the extension axiom. Let $\alpha \in I$, and let $\phi, \psi$ denote the germs of $p_{x}$ at $D(\alpha)$ and $\operatorname{Im}(\alpha)$, respectively. By the definition of $p_{x}$,

$$
p_{x}(D(\beta))=p_{x}(\operatorname{Im}(\beta)) \text { for all } \beta \in I \text {. }
$$

(7) For basic terminology pertaining to categories, cf. Cartan and Eilenberg [1]. 
Let $u$ be a local map such that $\alpha \in|u| \subset I$. It follows by (1.6) that $p_{x}(u(x))=$ $p_{x}(x)$ for all $x$ in the domain of $u$. Hence $\psi \alpha=\phi$, as was to be shown. The map generated by $\left|p_{\mathscr{X}}\right|$ will be referred to as the basic map of $\mathscr{X}$, and $\mathscr{I}(\hat{X})$ will be called the basic space of $\mathscr{X}$.

Proposition 6. Let $P$ denote the function which assigns to each object its basic map. There exists a unique (covariant) functor $\mathscr{P}: \mathscr{C} \rightarrow \mathscr{C}$ such that

(i) $\mathscr{P}(\mathscr{X})$ is the basic space of $\mathscr{X}$, for all objects $\mathscr{X}$;

(ii) $P$ is a morphism from the identity functor of $\mathscr{C}$ to $\mathscr{P}$.

To prove the proposition, we will first show that given a map $F: \mathscr{X} \rightarrow \mathscr{X}^{\prime}$, where $\mathscr{X}=(X, I)$ and $\mathscr{X}^{\prime}=\left(X^{\prime}, I^{\prime}\right)$ are objects, there exists a unique continuous function $\hat{F}: \hat{X} \rightarrow \hat{X}^{\prime}$ such that

$$
P\left(\mathscr{X}^{\prime}\right) \circ F=|\hat{F}| \circ P(\mathscr{X}) .
$$

We first establish uniqueness of $\hat{F}$ by considering an arbitrary point $\xi \in \hat{\mathscr{X}}$ and a germ $\phi \in F$ such that $p_{x}(D(\phi))=\xi$. One concludes by (1.7) that

$$
\hat{F}(\xi)=p_{\mathscr{X}^{\prime}}(\operatorname{Im}(\phi)),
$$

proving uniqueness. We will show next that formula (1.8) defines a function $\hat{F}$. For let $\psi \in F$ be a second germ such that $p_{x}(D(\psi))=\xi$. Then there exist $\alpha, \beta \in I$ such that $D(\alpha)=D(\phi), D(\beta)=D(\psi)$ and $\operatorname{Im}(\alpha)=\operatorname{Im}(\beta)$. Since $D(F)=X$, there exists a germ $\chi \in F$ such that $D(\chi)=\operatorname{Im}(\alpha)$. By the extension axiom for maps, there exist $\alpha^{\prime}, \beta^{\prime} \in I^{\prime}$ such that $\beta^{\prime} \phi=\alpha^{\prime} \chi \alpha$. We conclude by (1.6) that

$$
p_{x}(\operatorname{Im}(\phi))=p_{\mathscr{X}}(\operatorname{Im}(\chi))
$$

Similarly,

$$
p_{x}(\operatorname{Im}(\psi))=p_{x}(\operatorname{Im}(\chi))
$$

This implies that $\hat{F}$ is well-defined by formula (1.8).

The verification that $\hat{F}$ is continuous proceeds as follows. Let $U^{\prime}$ be an open subset of $\hat{\mathscr{X}}^{\prime}$, and let $V^{\prime}=p_{x^{\prime}}^{-1}\left(U^{\prime}\right)$. Clearly $V^{\prime}$ is open in $X^{\prime}$. Let $V$ denote the set of all points $x \in X$ for which there exists a germ $\phi \in F$ with $D(\phi)=x$ and $\operatorname{Im}(\phi) \in V^{\prime}$. One sees easily that $V$ is open, and that $p_{x}(V)=\hat{F}^{-1}\left(U^{\prime}\right)$. To prove that $\hat{F}^{-1}\left(U^{\prime}\right)$ is open, it therefore suffices to show that $V=p_{x}^{-1}\left(p_{x}(V)\right)$. Clearly $V \subset p_{\mathscr{X}}^{-1}\left(p_{x}(V)\right)$. Conversely, given $x \in p_{\mathscr{X}}^{-1}\left(p_{x}(V)\right)$, there exists a point $y \in V$ such that $p_{x}(x)=p_{x}(y)$. Hence there must exist $\alpha, \beta \in I$ with $D(\alpha)=x, D(\beta)=y$ and $\operatorname{Im}(\alpha)=\operatorname{Im}(\beta)$. Since $D(\beta) \in V$, there exists a germ $\phi \in F$ such that $D(\beta)=D(\phi)$ and $\operatorname{Im}(\phi) \in V^{\prime}$. There also exist germs $\chi, \psi \in F$ with $D(\chi)=\operatorname{Im}(\alpha)$ and $D(\psi)=D(\alpha)$. We now observe that the additional germs indicated in the following diagram exist in $I^{\prime}$ by the extension axioms, and render the diagram commutative. 


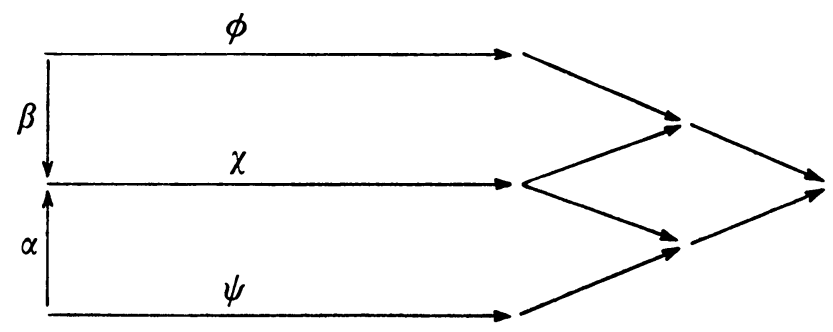

We conclude that

$$
p_{x}(\operatorname{Im}(\psi))=p_{x}(\operatorname{Im}(\phi)),
$$

and therefore that $x \in V$. This shows that $\hat{F}$ is continuous.

Now let $\mathscr{P}$ denote the function which to every object $\mathscr{X}$ assigns its basic space, and to every map $F: \mathscr{X} \rightarrow \mathscr{X}^{\prime}$ assigns $|\hat{F}|$. One easily verifies that $\mathscr{P}: \mathscr{C} \rightarrow \mathscr{C}$ is a functor. It follows now by Equation (1.7) that $P$ is a morphism of the desired kind. Lastly, since $\hat{F}$ is unique, so is $\mathscr{P}$.

As a corollary to Proposition 6, one obtains

Proposition 7. An object is a quasi-space of type $C$ if and only if its basic map is an isomorphism.

We note that in one direction this assertion is entirely trivial. For if $P(\mathscr{X})$ is an isomorphism, then $\mathscr{X}$ is a quasi-space by definition. Conversely, if $\mathscr{X}$ is a quasispace of type $C$, then there exists an object $\mathscr{X}^{\prime}$ in $\mathbb{C}$ and an isomorphism $F: \mathscr{X} \rightarrow \mathscr{X}^{\prime}$. Since $\mathscr{P}$ is a functor, $\mathscr{P}(F)$ is likewise an isomorphism. Moreover, it is easy to see that $P\left(\mathscr{X}^{\prime}\right)$ is an isomorphism, $\mathscr{X}^{\prime}$ being in $\mathbb{C}$. Since $P$ is a morphism, one has $\mathscr{P}(F) \circ P(\mathscr{X})=P\left(\mathscr{X}^{\prime}\right) \circ F$, implying that $P(\mathscr{X})$ is an isomorphism.

We will now show how the categories and subcategories previously considered can be regarded in a natural way as local categories and local subcategories in the sense of Cartan and Eilenberg [1]. We recall that a local category $\mathscr{A}$ consists of

(i) a category (which we also denote by $\mathscr{A}$ );

(ii) a (covariant) functor $L: \mathscr{A} \rightarrow C$;

(iii) a function which to every object $\mathscr{A}$ in $A$ and every open set $U \subset L(A)$

assigns (1) an object $A \mid U$ in $A$, and (2) a map $i_{A} \mid U$ in $\mathscr{A}$;

these data being subject to certain axioms. In order to regard $C$ itself as a local category, one takes $L$ to be the identity functor, $X \mid U$ to be $U$ and $i_{X} \mid U$ to be the inclusion map from $U$ to $X$. In the sequel, $C$ will often be regarded as a local category, without further explanation. To define a local category structure on $\mathscr{C}$, we note that $\mathscr{P}$ may be regarded as a functor from $\mathscr{C}$ to $\mathfrak{C}$. One can therefore identify the isomorphic categories $C$ and $\mathscr{C}$, and take $L$ to be $\mathscr{P}$. Moreover, given an object $\mathscr{X}=(X, I)$ and an open subset $U \subset \mathscr{P}(\mathscr{X})$, we take $\mathscr{X} \mid U=\left(V, I \cap G_{V}, V\right)$ and $i_{\mathscr{X}} \mid U=I \cap G_{V, V}$, where $V=p_{\mathscr{X}}^{-1}(U)$. Clearly $\mathscr{X} \mid U$ is again an object, and 
$i_{\mathscr{X}} \mid U$ is a map from $\mathscr{X} \mid U$ to $U$. By means of straightforward verifications, one now establishes the following result:

Proposition 8. The category $\mathscr{C}$, together with the additional structure defined above, constitutes a local category. Let $C_{0}$ be a local subcategory of $C$. The inclusion functors between $C_{0}, C, \mathscr{C}_{0}$ and $\mathscr{C}$ are strictly local.

2. Extension of sheaf-valued functors. 2.1. Preliminaries. In this section we shall be concerned with the problem of extending a sheaf-valued functor $S$, defined on a given local subcategory $C_{0}$ of $C$, to the corresponding object category $\mathscr{C}_{0}$. To make this precise, one could define a local category $\mathscr{F}$ of sheaves and regard $S$, together with its extension, as strictly local $\mathscr{F}$-valued functors. It will be substantially simpler and more economical, however, to obviate the category $\mathscr{F}$ by introducing the concept of sheaves on a local category. In this languagewhich will presently be explained $-S$ becomes a sheaf on $C_{0}$, and its extension will be a sheaf on $\mathscr{C}_{0}$.

Given a topological space $X$, we will let $c(X)$ denote the category consisting of all open subsets of $X$, together with their inclusion maps. By a (set-valued) presheaf on $X$ we shall understand a contravariant functor $G$ from $c(X)$ to the category $E$ of all sets and functions. Following Godement [3], $G$ will be called a sheaf on $X$ if the following two conditions are satisfied:

(i) Let $\left(U_{j}\right)_{j \in J}$ be an arbitrary family of open subsets of $X$, having union $U$. If $\sigma, \sigma^{\prime} \in G(U)$ and $G\left(i_{U} \mid U_{j}\right) \sigma=G\left(i_{U} \mid U_{j}\right) \sigma^{\prime}$ for all $j \in J$, then $\sigma=\sigma^{\prime}$.

(ii) Let $\left(U_{j}\right)_{j \in J}$ and $U$ be given as before. If $\sigma_{j} \in G\left(U_{j}\right)$ for $j \in J$ and $G\left(i_{U_{j}} \mid\right.$ $\left.U_{i} \cap U_{j}\right) \sigma_{j}=G\left(i_{U_{i}} \mid U_{i} \cap U_{j}\right) \sigma_{i}$ for all $i, j \in J$ with $U_{i} \cap U_{j} \neq \varnothing$, then there exists a $\sigma=G(U)$ such that $G\left(i_{U} \mid U_{j}\right) \sigma=\sigma_{j}$ for all $j \in J$.

We note for future reference that with every sheaf $G$ on $X$ there is associated an espace étalé in $X$, which will be denoted by $\mathscr{E}(G)$, and a natural projection $\pi: \mathscr{E}(G) \rightarrow X$. The stalk $\pi^{-1}(x)$ over a point $x \in X$ will be denoted by $G(x)$. If $U$ is an open subset of $X$, a continuous function $\sigma: U \rightarrow \mathscr{E}(G)$ is called a section over $U$ if $\sigma(x) \in G(x)$ for all $x \in U$. It is well known that elements of the set $G(U)$ may be canonically identified with sections over $U$. This identification will henceforth be understood. The restriction of $G$ to an open subset $U \subset X$ will be denoted by $G \mid U$.

Now let $\mathscr{A}$ be a local category, and let $A$ be an object in $\mathscr{A}$. We define a (covariant) functor $T_{A}: c(L(A)) \rightarrow \mathscr{A}$ (where $L: \mathscr{A} \rightarrow C$ is given by the local category structure of $\mathscr{A})$ as follows: Given an open subset $U \subset L(A), T_{A}(U)=A \mid U$, and given an open subset $V \subset U, T_{A}\left(i_{U} \mid V\right)=i_{A \mid U} \mid V$. The functorial character of $T_{A}$ is immediate. We now define a sheaf on $\mathscr{A}$ to be a contravariant functor $S: \mathscr{A} \rightarrow E$ such that, for every object $A$ in $\mathscr{A}, S \circ T_{A}$ is a sheaf on $L(A)$. An arbitrary contravariant functor $S: \mathscr{A} \rightarrow E$ will be called a presheaf on $\mathscr{A}$.

As may be expected, one finds that a presheaf $S$ on $\mathscr{A}$ generates a sheaf $S^{*}$ by 
means of a natural construction, which we proceed to describe. Given an object $A$ in $\mathscr{A}$, we let $\mathscr{F}_{A}$ denote the sheaf on $L(A)$ generated by the presheaf $S \circ T_{A}$. One can now take $S^{*}(A)$ to be the set $\mathscr{F}_{A}(L(A))$ of global sections. Given a map $F: A \rightarrow A^{\prime}$ in $\mathscr{A}$, we consider a section $\sigma^{\prime} \in S^{*}\left(A^{\prime}\right)$, a point $x \in L(A)$, and we let $x^{\prime}=L(F) x$. One now recalls that the stalk $\mathscr{F}_{A^{\prime}}\left(x^{\prime}\right)$ is a direct limit, and that consequently $\sigma^{\prime}\left(x^{\prime}\right)$ is a coset. We choose an arbitrary member $a^{\prime}$ in $\sigma^{\prime}\left(x^{\prime}\right)$. We recall that $a^{\prime}$ must belong to $S\left(A^{\prime} \mid U^{\prime}\right)$, where $U^{\prime}$ is an open neighborhood of $x^{\prime}$. Let $U$ denote the inverse image of $U^{\prime}$ under $L(F)$, and let $G=U^{\prime}|F| U\left({ }^{8}\right)$. Then $S(G) a^{\prime}$ belongs to $S(A \mid U)$, and determines a coset $\sigma(x) \in \mathscr{F}_{A}(x)$. It is not difficult to verify that $\sigma(x)$ is independent of our choice of $a^{\prime}$. Thus $\sigma^{\prime}$ may be pulled back to a section $\sigma \in S^{*}(A)$, which we denote by $S^{*}(F) \sigma^{\prime}$. This completes the definition of $S^{*}$.

Proposition 9. Let $S: \mathscr{A} \rightarrow E$ be a presheaf on $\mathscr{A}$. Then $S^{*}: \mathscr{A} \rightarrow E$ is a sheaf on $\mathscr{A}$. Moreover, there exists a canonical morphism $S_{*}$ from $S$ to $S^{*}$.

In the first place, it is immediate from our construction that $S^{*}$ is a contravariant functor on $\mathscr{A}$, i.e., a presheaf on $\mathscr{A}$. It remains therefore to show that $S^{*} \circ T_{A}$ is a sheaf on $L(A)$ for every object $A$ in $\mathscr{A}$. Let $A$ be such an object and $U$ an open subset of $L(A)$. Since $S \circ T_{A \mid U}$ is the restriction of $S \circ T_{A}$ to $c(U)$, one sees that $\mathscr{F}_{A \mid U}$ may be canonically identified with the restriction of $\mathscr{F}_{A}$ to $U$. Hence

$$
S^{*}(A \mid U)=\mathscr{F}_{A \mid U}(U)=\mathscr{F}_{A}(U) .
$$

It is now easily verified that

$$
S^{*}\left(i_{A} \mid U\right)=\mathscr{F}_{A}\left(i_{L(A)} \mid U\right) .
$$

Consequently $S^{*} \circ T_{A}$ equals $\mathscr{F}_{A}$, which is a sheaf on $L(A)$. This proves that $S^{*}$ is a sheaf on $\mathscr{A}$. Given an object $A$ in $\mathscr{A}$, we take $S_{*}(A)$ to be the canonical map from $S(A)$ to $S^{*}(A)$. It is trivial to verify that $S_{*}$ is a morphism from $S$ to $S^{*}$.

In the sequel we shall be concerned with a sheaf $S$ on a local subcategory $C_{0}$ of $C$. Associated with such a sheaf, one obtains certain stalk maps which we will now define. We consider two spaces $X, X^{\prime}$ in $C_{0}$, a germ $\phi \in G_{X, X^{\prime}}^{0}$, and a point $q$ belonging to the stalk $S \circ T_{X^{\prime}}(\operatorname{Im}(\phi))$. There exist open neighborhoods $U$ of $D(\phi)$ and $U^{\prime}$ of $\operatorname{Im}(\phi)$, together with a map $f: U \rightarrow U^{\prime}$ in $C_{0}$ and a section $\sigma^{\prime} \in S\left(U^{\prime}\right)$, such that $\phi \in|f| \subset G_{X, X^{\prime}}^{0}$ and $\sigma^{\prime}(\operatorname{Im}(\phi))=q$. One can now pull $\sigma^{\prime}$ back to a section $\sigma \in S(U)$ by applying $S(f)$. The point $\sigma(D(\phi))$, moreover, depends only on $\phi$ and $q$, as is easily checked. Thus to every germ $\phi \in G_{X, X^{\prime}}^{0}$, one can assign a function

$$
\phi_{S}^{*}: S \circ T_{X^{\prime}}(\operatorname{Im}(\phi)) \rightarrow S \circ T_{X}(D(\phi)) .
$$

(8) For this special notation, cf. Cartan and Eilenberg [1, p. 20]. 
It is clear that if $\phi \in G_{X, X^{\prime}}^{0}, \psi \in G_{X^{\prime}, X^{\prime \prime}}^{0}$ and $\psi \phi \in G_{X, X^{\prime \prime}}^{0}$, then $(\psi \phi)_{S}^{*}=\phi_{S}^{*} \circ \psi_{S}^{*}$.

2.2. The CANONICAL EXTENSION. Let $S$ be a sheaf on a local subcategory $C_{0}$ of $C$. We shall now describe a canonical extension of $S$ to a sheaf $\mathscr{S}$ on the (local) object category $\mathscr{C}_{0}$. Given a $\mathscr{C}_{0}$-object $\mathscr{X}=(X, I)$, we define an $S$-section over $\mathscr{X}$ to be a section $\sigma \in S(X)$ such that $\sigma(D(\alpha))=\alpha_{S}^{*} \sigma(\operatorname{Im}(\alpha))$ for all $\alpha \in I_{0}$. We take $\mathscr{S}(\mathscr{X})$ to be the set of all $S$-sections over $\mathscr{X}$. It remains to define the action of $\mathscr{S}$ on an arbitrary $\mathscr{C}_{0}$-map $F: \mathscr{X} \rightarrow \mathscr{X}^{\prime}$, where $\mathscr{X}=(X, I)$ and $\mathscr{X}^{\prime}=\left(X^{\prime}, I^{\prime}\right)$ are $\mathscr{C}_{0}$-objects. Thus one must show that an arbitrary $S$-section $\sigma^{\prime}$ over $\mathscr{X}^{\prime}$ can be pulled back by $F$ to an $S$-section $\sigma$ over $\mathscr{X}$. Given an open subset $U \subset X$ and a map $f: U \rightarrow X^{\prime}$ with $|f| \subset F_{0}$, we define a section $\sigma_{f} \in S(U)$ by the formula $\sigma_{f}=S(f) \sigma^{\prime}$. Now let $f, g: X \rightarrow X^{\prime}$ be two local maps, defined on open neighborhoods of $x \in X$, such that $|f|,|g| \subset F_{0}$. We will show that $\sigma_{f}(x)=\sigma_{g}(x)$. Let $\phi, \psi$ denote the germs of $f, g$ at $x$, respectively. By the extension axiom for object maps, there exist germs $\alpha^{\prime}, \beta^{\prime} \in I^{\prime}$ such that $\alpha^{\prime} \phi=\beta^{\prime} \psi$. This leads to the commutative diagram

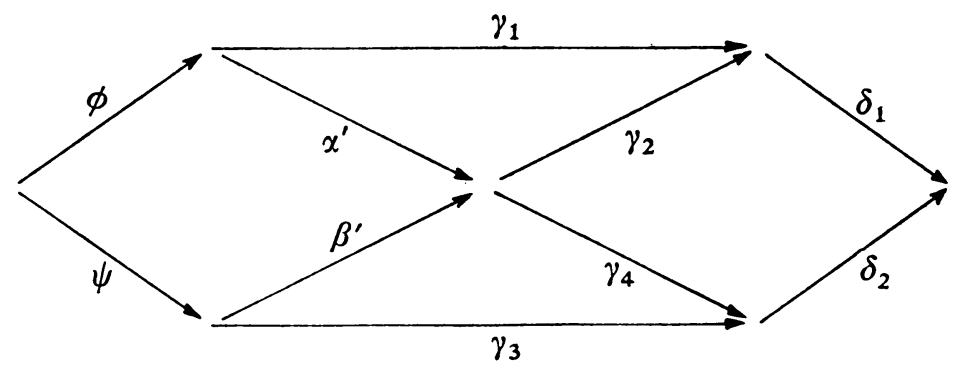

where $\gamma_{i}$ and $\delta_{j}$ are germs in $I_{0}^{\prime}$. Existence of the former is assured by Proposition 1 , and of the latter by the extension axiom for objects. Hence $\alpha \phi=\beta \psi$, where $\alpha=\delta_{1 \gamma_{1}}$ and $\beta=\delta_{2 \gamma_{3}}$. Since $I^{\prime}$ and $G_{X^{\prime}, X^{\prime}}^{0}$ are both closed under multiplication, so is their intersection, and consequently $\alpha, \beta \in I_{0}^{\prime}$. Therefore $\alpha \phi \in F_{0}$, and

$$
\sigma_{f}(x)=\phi_{S}^{*} \sigma^{\prime}(f(x))=\phi_{S}^{*} \alpha_{S}^{*} \sigma^{\prime}(\operatorname{Im}(\alpha))=(\alpha \phi)_{S}^{*} \sigma^{\prime}(\operatorname{Im}(\alpha))
$$

This equation, together with the corresponding one for $\sigma_{g}(x)$, implies that $\sigma_{f}(x)=\sigma_{g}(x)$. We may now conclude by axiom (ii) for sheaves that there exists a unique section $\sigma \in S(X)$ which agrees locally with $\sigma_{f}$ for all $f \in F$. Moreover, for every germ $\alpha \in I_{0}$, there exists a germ $\phi \in F_{0}$ such that $\phi \alpha \in F_{0}$. Therefore

$$
\sigma(D(\alpha))=(\phi \alpha)_{S}^{*} \sigma^{\prime}(\operatorname{Im}(\phi))=\alpha_{S}^{*} \phi_{S}^{*} \sigma^{\prime}(\operatorname{Im}(\phi))=\alpha_{S}^{*} \sigma(\operatorname{Im}(\alpha)),
$$

showing that $\sigma$ is an $S$-section over $\mathscr{X}$. We may consequently define $S(F)$ to be the function from $S\left(\mathscr{X}^{\prime}\right)$ to $S(\mathscr{X})$ which takes $\sigma^{\prime}$ to $\sigma$.

TheOREM 2. $\mathscr{S}: \mathscr{C}_{0} \rightarrow E$ is a sheaf. Moreover, $\mathscr{S}$ extends $S$. 
We will prove first that $\mathscr{S}$ is a functor. Let $\mathscr{X}=(X, I)$ be an object in $\mathscr{C}_{0}$ and $f: U \rightarrow X$ a map such that $|f| \subset I_{0}$. Given an $S$-section $\sigma$ over $\mathscr{X}$, one can define $\sigma_{f}$ as before. For an arbitrary germ $\alpha \in|f|$,

$$
\sigma_{f}(D(\alpha))=\alpha_{s}^{*} \sigma(\operatorname{Im}(\alpha))=\sigma(D(\alpha)) \text {. }
$$

Consequently $\mathscr{S}(I)$ is the identity map of $\mathscr{S}(\mathscr{X})$. Now let $F: \mathscr{X} \rightarrow \mathscr{X}^{\prime}$ and $F^{\prime}: \mathscr{X}^{\prime} \rightarrow \mathscr{X}^{\prime \prime}$ be maps in $\mathscr{C}_{0}$, where $\mathscr{X}=(X, I), \mathscr{X}^{\prime}=\left(X^{\prime}, I^{\prime}\right)$ and $\mathscr{X}^{\prime \prime}=\left(X^{\prime \prime}, I^{\prime \prime}\right)$. Let $\sigma^{\prime \prime} \in \mathscr{S}\left(\mathscr{X}^{\prime \prime}\right)$, and let $\sigma^{\prime}=\mathscr{S}\left(F^{\prime}\right) \sigma^{\prime \prime}, \sigma=\mathscr{S}(F) \sigma^{\prime}$ and $\hat{\sigma}=\mathscr{S}\left(F^{\prime} \circ F\right) \sigma^{\prime \prime}$. If $x$ is an arbitrary point of $X$, there exist germs $\phi \in F_{0}$ and $\psi \in F_{0}^{\prime}$ such that $D(\phi)=x$ and $\psi \phi \in\left(F^{\prime} \circ F\right)_{0}$. Then

$$
\hat{\sigma}(x)=(\psi \phi)_{S}^{*} \sigma^{\prime \prime}(\operatorname{Im}(\psi))=\phi_{S}^{*} \psi_{S}^{*} \sigma^{\prime \prime}(\operatorname{Im}(\beta))=\phi_{S}^{*} \sigma^{\prime}(\operatorname{Im}(\phi))=\sigma(x),
$$

showing that $\mathscr{S}\left(F^{\prime} \circ F\right)=\mathscr{S}(F) \circ \mathscr{S}\left(F^{\prime}\right)$. We have thus proved that $\mathscr{S}$ is a contravariant functor.

If $\mathscr{X}=(X, I)$ is an object in $\mathscr{C}_{0}, \mathscr{S} \circ T_{\mathscr{X}}$ is now a contravariant functor from $c(\mathscr{P}(\mathscr{X}))$ to $E$. We will show next that $\mathscr{S} \circ T_{\mathscr{X}}$ is in fact a sheaf on $\mathscr{P}(\mathscr{X})$, proving thus that $\mathscr{S}$ is a sheaf on $\mathscr{C}_{0}$. Let $\left(U_{j}\right)_{j \in J}$ be a family of open subsets of $\mathscr{P}(\mathscr{X})$, having union $U$, and let $\sigma, \bar{\sigma} \in \mathscr{S}(\mathscr{X} \mid U)$ be given such that

$$
\mathscr{S}\left(i_{x \mid U} \mid U_{j}\right) \sigma=\mathscr{S}\left(i_{x \mid U} \mid U_{j}\right) \bar{\sigma}
$$

for all $j \in J$. We observe that $\mathscr{S}(\mathscr{X} \mid U) \subset S(V)$, where $V=p_{\mathscr{X}}^{-1}(U)$, and that $\sigma, \bar{\sigma}$ may consequently be regarded as sections $\sigma, \bar{\sigma}: V \rightarrow \mathscr{E}\left(S \circ T_{X}\right)$. One also sees that $\mathscr{S}\left(i_{\mathscr{X} \mid U} \mid U_{j}\right)$ is just the restriction of $S\left(i_{V} \mid V_{j}\right)$ to $\mathscr{S}(\mathscr{X} \mid U)$, where $V_{j}=p_{\mathscr{X}}^{-1}\left(U_{j}\right)$. Consequently Equation (2.2) implies

$$
S\left(i_{V} \mid V_{j}\right) \sigma=S\left(i_{V} \mid V_{j}\right) \bar{\sigma}
$$

for all $j \in J$. Since $S \circ T_{X}$ is a sheaf on $X$, we may conclude by axiom (i) for sheaves that $\sigma=\bar{\sigma}$. Hence $\mathscr{S}_{\circ} T_{\mathscr{X}}$ likewise satisfies axiom (i).

We suppose now that elements $\sigma_{j} \in \mathscr{S}\left(\mathscr{X} \mid U_{j}\right)$ are given such that

$$
\mathscr{S}\left(i_{\mathscr{X} \mid U_{j}} \mid U_{j} \cap U_{k}\right) \sigma_{j}=\mathscr{S}\left(i_{\mathscr{X} \mid U_{k}} \mid U_{j} \cap U_{k}\right) \sigma_{k}
$$

for all $j, k \in J$ with $U_{j} \cap U_{k} \neq \varnothing$. This means again that

$$
S\left(i_{V_{j}} \mid V_{j} \cap V_{k}\right) \sigma_{j}=S\left(i_{V_{k}} \mid V_{j} \cap V_{k}\right) \sigma_{k}
$$

for all $j, k \in J$ with $V_{j} \cap V_{k} \neq \emptyset$. By axiom (ii) for sheaves, there exists a section $\sigma \in S(V)$ which agrees locally with $\sigma_{j}$ for all $j \in J$. It thus remains to show that $\sigma$ is an $S$-section over $\mathscr{X} \mid U$. For this purpose we consider an arbitrary germ $\alpha \in I_{0} \cap G_{V, V}^{0}$. Then $D(\alpha) \in V_{j}$ for some $j \in J$, which implies $\operatorname{Im}(\alpha) \in V_{j}$. Since $\sigma_{j}$ is an $S$-section over $\mathscr{X} \mid U_{j}$ and $\sigma$ agrees locally with $\sigma_{j}$, one finds that $\sigma(D(\alpha))=\alpha_{s}^{*} \sigma(\operatorname{Im}(\alpha))$. Consequently $\sigma \in \mathscr{S}(\mathscr{X} \mid U)$, as was to be proved. 
The remaining assertion of Theorem 2 , i.e., that $\mathscr{S}$ extends $S$, is an immediate consequence of our construction.

2.3. Cohomology theories. Let $A$ be a commutative ring, and let $\mathscr{M}_{A}$ denote the category of $A$-modules and $A$-homomorphisms. A sheaf $\mathscr{F}: \mathscr{A} \rightarrow \mathscr{M}_{A}$, where $\mathscr{A}$ is a local category, will be called a sheaf of $A$-modules on $\mathscr{A}$.

Now let $S$ be a sheaf of $A$-modules on a local subcategory $C_{0}$ of $C$, and let $\mathscr{X}=(X, I)$ be an object in $\mathscr{C}_{0}$. It follows readily from the definition of $S$-section over $\mathscr{X}$ that $\mathscr{S}(\mathscr{X})$ is an $A$-submodule of $S(X)$. Similarly, if $F$ is a map in $\mathscr{C}_{0}$, one finds that $\mathscr{S}(F)$ is an $A$-homomorphism.

By a differential $A$-module, or simply $(d, A)$-module, we shall understand an $A$-module endowed with null-square endomorphism $d$. We will let $\mathscr{M}_{A}^{d}$ denote the category of $(d, A)$-modules and $(d, A)$-homomorphisms. The notion of $(d, A)$ sheaf on a local category can be defined in an obvious way, and one finds as before that if $S$ is a $(d, A)$-sheaf on $C_{0}$, its extension $\mathscr{S}$ will be a $(d, A)$-sheaf on $\mathscr{C}_{0}$. Thus we have

Proposition 10. If $S: C_{0} \rightarrow \mathscr{M}_{A}\left(\mathscr{M}_{A}^{d}\right)$ is a sheaf, then its extension $\mathscr{S}: \mathscr{C}_{0} \rightarrow E$ takes values in $\mathscr{M}_{A}\left(\mathscr{M}_{A}^{d}\right)$.

If $M$ is an $A$-module, we let $E_{M}$ denote the subcategory of $\mathscr{M}_{A}$ consisting only of $M$ and its identity map. If $\mathscr{A}$ is a category, there exists exactly one functor from $\mathscr{A}$ to $E_{M}$, which we denote by $M_{\mathscr{A}}$. A sheaf $\mathscr{F}: \mathscr{A} \rightarrow \mathscr{M}_{A}$ of $A$-modules on a local category $\mathscr{A}$ will be called trivial if there exists an $A$-module $M$ and a morphism $T: \mathscr{F} \rightarrow M_{\mathscr{A}}$ such that $T(\mathscr{X})$ is an $A$-isomorphism for every object $\mathscr{X}$ in $\mathscr{A}$.

Given a $(d, A)$-module $M$, we shall let $H(M)$ denote the cohomology module of $M$ (i.e., kernel of $d$ modulo the image of $d$ ). Moreover, given a homomorphism $f: M \rightarrow M^{\prime}$ of $(d, A)$-modules, we will let $H(f)$ denote the natural homomorphism from $H(M)$ to $H\left(M^{\prime}\right)$ induced by $f$. This defines a covariant functor $H: \mathscr{M}_{A}^{d} \rightarrow \mathscr{M}_{A}$.

Now let $C_{0}$ be a local subcategory of $C$. A sheaf $S: C_{0} \rightarrow \mathscr{M}_{A}^{d}$ may be called a cohomology theory on $C_{0}$ if the sheaf $(H \circ S)^{*}: C_{0} \rightarrow \mathscr{M}_{A}$ is trivial. Examples are (i) the sheaf of $C^{\infty}$ differential forms on the category of $C^{\infty}$ manifolds and $C^{\infty}$ maps, and (ii) the sheaf of Alexander-Spanier cochains on $C$. Given a cohomology theory $S$ on $C_{0}$, one can extend $S$ canonically to the sheaf $\mathscr{S}: \mathscr{C}_{0} \rightarrow \mathscr{M}_{A}^{d}$. One thus obtains the functor $H \circ \mathscr{S}: \mathscr{C}_{0} \rightarrow \mathscr{M}_{A}$, which extends the classical cohomology functor $H \circ S$. Furthermore, it is found that the sheaf $(H \circ \mathscr{S})^{*}$ : $\mathscr{C}_{0} \rightarrow \mathscr{M}_{A}$ is not trivial in general, as will appear from a simple example. One therefore obtains an essentially new cohomology functor $(H \circ \mathscr{S})^{*}$ on $\mathscr{C}_{0}$, together with the morphism $(H \circ \mathscr{S})_{*}: H \circ \mathscr{S} \rightarrow(H \circ \mathscr{S})^{*}$.

2.4. AN EXAMPLE. In most examples, a cohomology theory $S: C_{0} \rightarrow \mathscr{M}_{A}^{d}$ takes values in the category $\mathscr{G}_{A}^{d}$ of graded differential $A$-algebras. Then $\mathscr{S}$ will likewise take values in $\mathscr{G}_{A}$, and the functors $(H \circ \mathscr{S}),(H \circ \mathscr{S})^{*}$ take values in the 
category $\mathscr{G}_{A}$ of graded $A$-algebras. If $X$ is an object in $C_{0},(H \circ S)^{*}(X)$ will generally be the ring $A$ (graded in the obvious way).

Now let $C_{0}$ be the category of smooth (i.e., $C^{\infty}$ ) manifolds and smooth maps. Let $R$ denote the ring of real numbers and $S: C_{0} \rightarrow \mathscr{G}_{R}^{d}$ the sheaf of smooth differential forms on $C_{0}$. We shall give an example of an object $\mathscr{X}$ in $\mathscr{C}_{0}$ whose cohomology algebras $H \circ \mathscr{S}(\mathscr{X})$ and $(H \circ \mathscr{S})^{*}(\mathscr{X})$ are distinct and nontrivial. Let $Y$ denote the 1 -sphere (the real numbers $\bmod 2 \pi$ ), and let $X=R \times Y$. For every $a \in R$, we define a function $f_{a}: X \rightarrow X$ by the formula

$$
f_{a}(x, \theta)=(x+a, \theta) \text { for } x \in R \text { and } \theta \in Y \text {. }
$$

Then $\mathscr{X}=(X, I)$ is an object, where $I=\bigcup_{a \in R}\left|f_{a}\right|$. Moreover, since $I=I_{0}$, $\mathscr{X}$ belongs to $\mathscr{C}_{0}$.

Using the traditional notation, a differential form $\omega \in S(X)$ can be written as

$$
\omega=f_{1}+f_{2} d x+f_{3} d \theta+f_{4} d x d \theta
$$

where the $f_{i}$ are smooth functions of $(x, \theta)$, periodic in $\theta$. The differential $d \omega$ is given by

$$
d \omega=\frac{\partial f_{1}}{\partial x} d x+\frac{\partial f_{1}}{\partial \theta} d \theta+\left(\frac{\partial f_{3}}{\partial x}-\frac{\partial f_{2}}{\partial \theta}\right) d x d \theta .
$$

A form $\omega \in S(X)$ will belong to $\mathscr{S}(\mathscr{X})$ if $S\left(f_{a}\right) \omega=\omega$ for every $a \in R$. This is the case if and only if the coefficients $f_{i}$ are independent of $x$. Hence every form $\omega \in \mathscr{S}(\mathscr{X})$ is given by the expression (2.3), the coefficients $f_{i}$ being smooth periodic functions of $\theta$. One sees by (2.4) that $d \omega=0$ if and only if $f_{1}, f_{2}$ are constant. On the other hand, such a form will cobound in $\mathscr{S}(\mathscr{X})$ if and only if $f_{1}=f_{2}=0$ and

$$
\int_{0}^{2 \pi} f_{j}(\theta) d \theta=0, \quad j=3,4 .
$$

Since every periodic function can be uniquely represented as the sum of a constant and a function having zero average, it follows that every cocycle in $\mathscr{S}(\mathscr{X})$ can be uniquely represented in the form $\omega=\hat{\omega}+\bar{\omega}$, where $\bar{\omega}$ cobounds in $\mathscr{S}(\mathscr{X})$ and $\hat{\omega}$ is of the form

$$
\hat{\omega}=a_{1}+a_{2} d x+a_{3} d \theta+a_{4} d x d \theta,
$$

with $a_{i} \in R$. We have thus represented $(H \circ \mathscr{S})(\mathscr{X})$ as the subalgebra of $\mathscr{P}(\mathscr{X})$ consisting of all forms $\hat{\omega}$ given by (2.5).

The basic space $\mathscr{P}(\mathscr{X})$ may be canonically identified with $Y, p_{\mathscr{X}}: R \times Y \rightarrow Y$ being the natural projection. Let $U$ be an open interval of $Y$ (distinct from $Y$ ). A differential form $\omega \in S(R \times U)$ is represented by (2.3), the coefficients $f_{i}$ being now arbitrary smooth functions on $R \times U$. One sees again that $\omega \in \mathscr{S}(\mathscr{X} \mid U)$ if and only if the coefficients are independent of $x$. The cocycles are now charac- 
terized by the condition that $f_{1}, f_{2}$ be constant. Moreover, a cocycle cobounds in $\mathscr{S}(\mathscr{X} \mid U)$ if and only if $f_{1}=f_{2}=0$. Hence every $\omega \in \mathscr{S}(\mathscr{X} \mid U)$ can be uniquely represented in the form $\omega=\hat{\omega}+\bar{\omega}$, where $\bar{\omega}$ cobounds in $\mathscr{S}(\mathscr{X} \mid U)$ and

$$
\hat{\omega}=a_{1}+a_{2} d x
$$

with $a_{i} \in R$. Thus $H \circ \mathscr{S}(\mathscr{X} \mid U)$ has been represented as a subalgebra of $H \circ \mathscr{S}(\mathscr{X})$.

Now let $U, V$ be open subintervals of $Y$, with $V \subset U$. In terms of the given representations, $H \circ \mathscr{S}\left(i_{\mathscr{X} \mid \cup} \mid V\right)$ is just the identity. Since the open intervals $U$ constitute a basis for the topology of $Y$, it follows that we may take $(H \circ \mathscr{S})^{*}(\mathscr{X})$ to be $(H \circ \mathscr{S})(\mathscr{X} \mid U)$ for some particular $U$. Then $(H \circ \mathscr{S})_{*}(\mathscr{X})$ is the function which takes a form (2.5) to the form (2.6).

We have thus established that the algebra $(H \circ \mathscr{S})^{*}(\mathscr{X})$ is nontrivial, and that $(H \circ \mathscr{S})_{*}(\mathscr{X})$ is not one-to-one.

\section{REFERENCES}

1. H. Cartan and S. Eilenberg, Foundation of fibre bundles, Symposium Internacional de Topología Algebraica, Mexico, 1958.

2. Y.H. Clifton and J. W. Smith, The category of topological objects, Proc. Nat. Acad. Sci. U.S.A. 47 (1961), 190-195.

3. R. Godement, Théorie des faisceaux, Hermann, Paris, 1958.

Massachusetts Institute of Technology, CAMbridge, MassachusetTS

UNIVERSITY OF CALIFORNIA, Los Angeles, California 